\title{
Efficient message exchange protocols exploiting state-of-the-art PHY layer
}

\author{
João S. Lemos ${ }^{1,2}$, Francisco A. Monteiro ${ }^{1,3^{*}}$ (D), Ivo Sousa ${ }^{1,2}$ and Filipe E. Ferreira ${ }^{1}$
}

\begin{abstract}
The paper focuses on the two-way relay channel (TWRC) and the multi-way wireless network with three terminals, where all three want to exchange or share data and have to do that with the help of a relay. This paper shows how it is possible to significantly decrease the number of time slots required to exchange messages between terminals in networks based on time-division multiple access (TDMA), by taking into consideration new techniques at the physical (PHY) layer. The paper considers a PHY layer where physical-layer network coding (PLNC), multiple-input multiple-output (MIMO), and in-band "full-duplex" (IBFD) with loopback interference cancellation are all integrated, so that it is possible to significantly increase the overall throughput of the network. This is entirely attained by transferring the burden from the time domain to the spatial domain, via spatial multiplexing and by simultaneously resorting to non-orthogonal multiple access, which is the consequence of using both PLCN and IBFD. For the TWRC, it is shown that, if a massive MIMO relay is used, a simple lattice-based PLNC can be directly applied and, with typical IBFD interference cancellation amounts, a TWRC can effectively use only one time slot instead of the four needed when adopting the traditional TDMA exchange. In the case of the $Y$-network (i.e., with three terminals), a technique is presented that allows all the information exchange between terminals to be cut from the six time slots required in TDMA to only one time slot, provided that the information packets are not too short. The error performance of these systems is measured by means of simulation using MIMO Rayleigh fading channels.
\end{abstract}

Keywords: Y-network, Two-way relay channel, MIMO, Massive MIMO, Full-duplex, Physical-layer network coding, Packets exchange, Interference suppression

\section{Introduction}

In wireless networks where a relay node intervenes, the traditional way of exchanging messages (symbols or packets) between two or more terminals either involves time-domain multiplexing (TDMA) or dedicated frequency-domain disjoint channels, at the expense of high bandwidth inefficiency. Interference in wireless networks has been until recently considered a central problem and has been mostly avoided in order to facilitate transmissions between the nodes of a network. For that reason, simultaneous transmissions are treated carefully, so that the interference between users is strictly avoided,

\footnotetext{
*Correspondence: francisco.monteiro@|x.it.pt

This paper was partially presented at the 18th IEEE Region 8 Mediterranean Electrotechnical Conference (MELECON 2016), Limassol, Cyprus, 18-20 April, 2016, and at the 9th IEEE Sensor Array and Multichannel Signal Processing Workshop (IEEE SAM 2016), Rio de Janeiro, Brazil, 10-13 July, 2016. ${ }^{1}$ Instituto de Telecomunicações, Lisbon, Portugal

${ }^{3}$ ISCTE_-Instituto Universitário de Lisboa, Lisbon, Portugal

Full list of author information is available at the end of the article
}

and this has been contributing to the limitation of the capacity of commercial networks. Wireless networks usually employ scheduling algorithms over the time and frequency resources so as to achieve the aforementioned goal. However, with a proper characterization of the interference, these resources may be more efficiently used, thus, enlarging the amount of information exchanged in wireless networks. For that reason, interference may be seen as nothing more than the superposition or sum of delayed and attenuated versions of the user's transmitted signals, and therefore, it can be "decoded", rather than entirely avoided.

Higher data rates and lower latencies are two central objectives when advancing wireless networks, but while the role of interference at the physical (PHY) layer of wireless networks has recently been profoundly re-thought with the emergence of new techniques to combat and exploit it in order to maximize the efficiency of the physical resources [1], most of these advances in the PHY layer 
of wireless communications have not yet been translated to the way that message exchange protocols make use of the PHY layer $[2,3]$.

Some of these recent developments in the PHY layer are (i) physical-layer network coding (PLNC), based on the idea that information packets can be superimposed and still recovered as long as the receiver knows part of the information that was superimposed; (ii) multiple-input multiple-output (MIMO) terminals and relays, which allow to boost the time-usage efficiency by transferring the burden from the time domain to the spatial domain, exploiting the spatial multiplexing permitted by having multiple antennas at the terminals; (iii) massive MIMO, where a very large number of antennas create an important channel orthogonality; and (iv) in-band "full-duplex" (IBFD) technology, which allows terminals and relays to transmit and receive at the same time in the same frequency band by applying several layers of loopback interference (LI) cancellation. In the case of IBFD, one very important line of research to further increase the sumrate of a system is the optimization of the transmission powers of the relay and terminals [4] or just the one at the relay [5]. All these techniques will be central in the next generation of the PHY layer of wireless communications [6], and Section 2 will overview each one of them. In fact, even the medium access control protocols can benefit much from redesigning the protocol taking into consideration multi-carrier modulations such as orthogonal frequency-division multiplexing (OFDM) [7].

This paper starts by looking at the two-way relay channel (TWRC), where two terminals, for some reason, cannot directly communicate (e.g., due to propagation obstacles or power limitations) and are forced to exchange data via a relay. It is well known that the number of time slots required to exchange the information between the two terminals can be brought down from four time slots to just two [8]. By deploying a relay using a massive array, a lattice-based PLNC scheme becomes possible and, by applying recently developed cancellation techniques for the self-interference, IBFD also becomes possible, allowing the scheme to be able to ultimately exchange information across the TWRC using only one time slot. Note that this contrasts with the four time slots that would be needed in a conventional TDMA-based TWRC. The paper considers the joint application of the three aforementioned technologies at the PHY layer. It should be highlighted that the orthogonal properties of having massive MIMO at the relay are conjugated with PLNC, allowing to increase the amount of information exchanged per channel use and also contributing to further cancel the $\mathrm{LI}$ at the relay. Furthermore, with massive MIMO, a simple orthogonal lattice-based PLNC scheme becomes possible, and the dependency of the system's performance on the number of antennas at the relay is assessed.
Secondly, the paper considers the extension to three terminals, which is sometimes named in the literature as the Y-channel $[9,10]$, and in this paper will be referred to as the Y-network. It comprises three terminals communicating with each other with the help of a relay and can be regarded as a generalized network model of the TWRC for three different users, when the terminals cannot physically establish direct connections among them and where each terminal has some information that wants to transmit to the other two. With the advent of MIMO, network coding, and later PLNC, it became possible to reduce the number of time slots required to exchange the information among all the terminals. To that end, two strategies have been proposed in $[11,12]$, and those reduced the six slots required in traditional TDMA to three slots and then to two slots only.

In this paper, IBFD is incorporated both at the terminals and at the relay together with PLNC and MIMO, in order to attain the maximum throughput in each of the two wireless network configurations described above. One will consider that some interference is suppressed at the physical layer, while some of it persists and impairs the layers above. The strategies proposed in this paper are able to, on average, reduce the communication stages to a single time slot per message exchanged. The error performance of the systems is determined by means of simulation using flat Rayleigh fading channels.

The paper is organized as follows. Section 2 gives an overview of the main PHY techniques that will be considered in the networks later assessed. Sections 3 and 4 respectively present the proposed strategies for the exchange of information in the TWRC and in the Y-network. The performance results obtained by simulation are provided in Section 5, which is followed by the conclusions in Section 6.

\section{Modern signal processing at the physical layer}

Several techniques have been put forward to increase the spectral efficiency of the PHY layer of wireless networks. Despite much effort on cross-layer optimization, there is still room to design new network protocols for packet exchange that leverage these opportunities being created at the physical layer. One can first point out that the long-held assumption that radios can only simultaneously transmit and receive in different frequency bands (i.e., imposing orthogonality in the frequency domain) has ended. This idea of splurging spectrum was until recently deemed necessary to avoid interference. The recent concept of IBFD communications makes use of the same frequency band to both transmit and receive data in wireless nodes, and it is expected to be incorporated in the upcoming wireless generation [6], providing a leap forward in terms of spectral efficiency. Full-duplex may ideally double a link's capacity or, equivalently, reduce by half the 
allocated frequency band, when it is compared with the current half-duplex or out-of-band "full-duplex" modes. However, since both frequency and time resources are used simultaneously, the limitations of IBFD operation arise from the existing self-interference, which reflects the leakage of the transceiver's outgoing signal to its reception side, a problem that is enhanced by the high power unbalance between both signals, hence, potentially causing inadmissible levels of interference that deteriorate the system's performance [13]. Self-interference must therefore be mitigated, and this is typically done at three different independent stages [14]. The first cancellation stage is performed within the wireless propagation domain, essentially by using passive techniques that can electromagnetically isolate signals. Then, analog radio circuits are employed at a broadband level to further reduce the self-interference signal power. These circuits create a delayed and phase-rotated version of the outgoing signal that is subtracted to the incoming one, aiming at tracking and simulating the effect of the channel [15]. Finally, the third (digital) stage is required in the signal processing domain in order to provide a fine mitigation of the residual interference still present after the first two steps [5].

Another cornerstone technology in 5G is the use of massive MIMO arrays (possibly employing hundreds of antennas) at the base stations and relays, which allows serving more users, i.e., increasing the overall system's capacity. Theoretically, using a $N \times N$ MIMO system can increase by $N$ the system throughput (for example, by taking advantage of a single-valued decomposition (SVD) of the channel and using adequate precoding) [16]. Massive MIMO upscales the attractiveness of MIMO by reducing noise, fading, and interference [17].

Finally, PLNC has emerged as a new way of thinking interference in multi-hop networks. The idea is to treat multi-user interference as a necessary effect, rather than avoid it by allocating different channel resources to different users $[18,19]$. PLNC applies the principle of network coding [20] taking in consideration the additive property of wireless channels and was simultaneous proposed in three independent works [21-23]. With a sufficient number of linear combinations of messages, it is possible to recover all the messages. Afterwards, a more practical approach to the problem emerged, which explores the capacity of a relay to decode a combination of symbol constellations $[24,25]$. Also, an information theoretic approach emerged, taking advantage of codebooks and lattice network coding [26, 27].

Table 1 presents a summary of the aforementioned PHY layer techniques, as well as related bibliography for further reading.

There is also the possibility of employing massive MIMO arrays in order to cope with the self-interference
Table 1 PHY layer techniques

\begin{tabular}{|c|c|c|}
\hline Technique & Goals & Bibliography \\
\hline IBFD & $\begin{array}{l}\text { To double the spectral } \\
\text { efficiency of a wireless } \\
\text { network by transmitting } \\
\text { and receiving data at the } \\
\text { same time and in the } \\
\text { same frequency band }\end{array}$ & {$[5,6,13-15,35,39,40,46-56]$} \\
\hline MIMO & $\begin{array}{l}\text { To provide transmit } \\
\text { beamforming, spatial } \\
\text { multiplexing, } \\
\text { space-division multiple } \\
\text { access and interference } \\
\text { alignment }\end{array}$ & {$[17,41,57-61]$} \\
\hline PLNC & $\begin{array}{l}\text { To use the channel itself } \\
\text { to help to perform a } \\
\text { linear combination of } \\
\text { messages that are then } \\
\text { forwarded }\end{array}$ & {$[8,18-21,23,25-27,62-65]$} \\
\hline
\end{tabular}

present in IBFD terminals. The orthogonality property of these large-scale channels allows a better level of mitigation in the self-interference component. In [28], it is proven that massive MIMO renders more resilience in terms of inter-pair interference, while also mitigating the self-interference effect. The authors proposed a zero-forcing (ZF) precoding and an extended regularized channel inversion that is proven to exploit and combine the advantages of massive MIMO systems and in-band "full-duplex" transmissions. In [29], filtering suppression (which takes advantage of MIMO systems to perform filtering) and time-domain cancellation (which subtracts an estimation of the interference from the received signal) are compared; the authors evaluated a bidirectional stream of communication and compared null-space projection schemes with time-domain cancellation having the same degrees of freedom. The methods are compared based on the achievable rates, and they concluded that timedomain cancellation have better achievable rate regions for the channel model considered. Additionally, they observed that antenna imbalance, i.e., having more antennas to transmit than receive or vice-versa, can improve suppression methods. In [30], the same authors presented a paper based on MIMO transmission links with loopback interference suppression decode-and-forward (DF) scheme, which means that the relay fully regenerates the digital signal.

Combining massive MIMO with an IBFD relay station may provide outstanding results. In [4], a multipair DF "full-duplex" relay that combines massive antenna array techniques is presented to mitigate the self-interference borne by the relay. Those authors propose a method where the relay station receives pilots to estimate the loopback channel and then processes the signal using ZF or maximum-ratio combining/maximum-ratio transmission 
(MRC/MRT) detection and precoding. The multipair of users are seen as a distributed multiple-input transmitting to the multiple-output relay. Thus, a linear ZF or MRC detection algorithm decodes the received signal. Since linear decoders can perform as well as non-linear ones with large arrays [31], the outgoing signal is precoded with a corresponding ZF or MRT and forwarded. The authors show that when the relay input and output antennas tend to infinity, the LI becomes orthogonal with the desired signal, perfectly canceling its undesired effect. Moreover, an optimization of the power allocation is developed, where the system energy efficiency (EE) is maximized, only subject to a given spectral efficiency and peak power. Nevertheless, their results assume perfect channel state information (CSI) for the large-scale fading components of the channels, and for that reason, interference will always be present, albeit, with a low power component.

Few works combining in-band "full-duplex" and PLNC exist in the literature. Zheng has proposed a system for the TWRC [32], where both the relay and the users have multiple antennas. An analog network coding (ANC) to forward information with a ZF constrain at the relay was proposed there, in order to attenuate the problem of self-interference. Moreover, the author presents power control to optimize the system's rates. Tedik and Kurt have presented a system that utilizes DF relaying based on maximum likelihood (ML) estimation of the XOR function for binary pulse shift keying (BPSK) [33]. The selfinterference from "full-duplex" transmissions at the nodes and at the relay are canceled with antenna separation at the propagation level and with time-domain cancellation at a digital level. Very recently, techniques mostly used in sensor fusion were applied to the TWRC with PLNC [34].

These ideas remain valid in the most simple lattices one can think of: the orthogonal lattices, which allow an orthogonal basis. In this paper, one will leverage on the fact that massive MIMO allows to naturally communicate over such an orthogonal structure, permitting a quite elegant application of lattice-based PLNC. As a step forward, this paper will then combine PLNC with inband "full-duplex" transmissions. As already mentioned, TDMA-based exchange of information in the TWRC is accomplished in four time slots. Network coding reduces this number to only three slots. PLNC further improves the exchange of information to two time slots. Finally, by incorporating IBFD, only one time slot may be used to sustain bidirectional streams of information. This could be the major step towards finding solutions to meet the requirements of future networks.

The paper considers a central relay with two or three users who want to send their message to all the other users in the network. The first case, to be described in Section 3, corresponds to the TWRC and the second to the Y-network, which will be described in Section 4. In both cases, the terminals transmit and receive simultaneously and in the same frequency band.

\section{The two-users case: the TWRC}

In the two-terminal case, the MIMO relay is considered to have a massive array, and one also considers that the two terminals operate a IBFD mode. Given that the system is symmetric, only the performance of one of the communication directions is assessed.

Consider that a terminal $\mathcal{A}$ and a terminal $\mathcal{B}$, both terminals with $N_{T}$ receive and $N_{T}$ transmit antennas, exchange information via a relay station $\mathcal{R}$, which is assumed to have $M_{R}>>N_{T}$ antennas to receive and $M_{T}=N_{T}$ antennas to transmit, as Fig. 1 depicts.

The received signals at each element at time slot $n$ of the system are expressed by:

$$
\begin{aligned}
\mathbf{y}_{\mathbf{R}}(n)= & \sqrt{p_{A}} \mathbf{H}_{\mathbf{A R}} \mathbf{x}_{\mathbf{A}}(n)+\sqrt{p_{B}} \mathbf{H}_{\mathbf{B R}} \mathbf{x}_{\mathbf{B}}(n) \\
& +\sqrt{p_{R}} k_{R} \mathbf{H}_{\mathbf{R} \mathbf{R}} \mathbf{x}_{\mathbf{R}}(n)+\mathbf{n}_{\mathbf{R}}(n), \\
\mathbf{y}_{\mathbf{A}}(n)= & \sqrt{p_{R}} \mathbf{H}_{\mathbf{R A}} \mathbf{x}_{\mathbf{R}}(n)+\sqrt{p_{A}} k_{A} \mathbf{H}_{\mathbf{A A}} \mathbf{x}_{\mathbf{A}}(n)+\mathbf{n}_{\mathbf{A}}(n),(2) \\
\mathbf{y}_{\mathbf{B}}(n)= & \sqrt{p_{R}} \mathbf{H}_{\mathbf{R B}} \mathbf{x}_{\mathbf{R}}(n)+\sqrt{p_{B}} k_{B} \mathbf{H}_{\mathbf{B B}} \mathbf{x}_{\mathbf{B}}(n)+\mathbf{n}_{\mathbf{B}}(n),(3)
\end{aligned}
$$

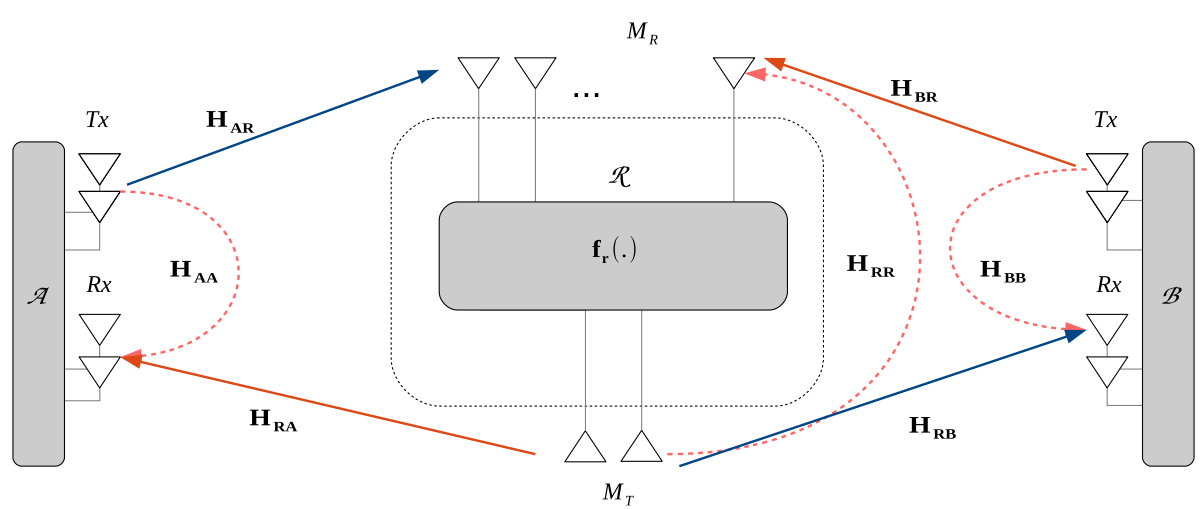

Fig. 1 Massive MIMO relay system for the FD-TWRC, for a large $M_{R}$ and for $M_{T}=N_{T}=2$ 
where $\mathbf{x}_{\mathbf{A}}(n), \mathbf{x}_{\mathbf{B}}(n)$ and $\mathbf{x}_{\mathbf{R}}(n)$ are the terminal $\mathcal{A}$, terminal $\mathcal{B}$ and relay transmit signals, respectively. Matrices $\mathbf{H}_{\mathrm{AR}} \in$ $\mathbb{C}^{M_{R} \times N_{T}}, \mathbf{H}_{\mathbf{B R}} \in \mathbb{C}^{M_{R} \times N_{T}}, \mathbf{H}_{\mathbf{R A}} \in \mathbb{C}^{N_{T} \times M_{T}}$ and $\mathbf{H}_{\mathbf{R B}} \in$ $\mathbb{C}^{N_{T} \times M_{T}}$ represent the channels from $\mathcal{A}$ and $\mathcal{B}$ to $\mathcal{R}$, and vice-versa. Matrices $\mathbf{H}_{\mathbf{A A}} \in \mathbb{C}^{N_{T} \times N_{T}}, \mathbf{H}_{\mathbf{B B}} \in \mathbb{C}^{N_{T} \times N_{T}}$ and $\mathbf{H}_{\mathbf{R R}} \in \mathbb{C}^{M_{R} \times M_{T}}$ represent the self-interference channels, while $\mathbf{n}_{\mathbf{A}}(n), \mathbf{n}_{\mathbf{B}}(n)$ and $\mathbf{n}_{\mathbf{R}}(n)$ account for the complex circularly symmetric Gaussian noise vectors. The transmit average power of the elements involved in the system are given by $p_{A}, p_{B}$ and $p_{R}$, respectively. Furthermore, the self-interference is mitigated through parameters $k_{A}$, $k_{B}$ and $k_{R}$, that translate the suppression levels, with respect to the situation without interference. The residual interference (i.e., the discrepancy between the estimated interference and the real interference) is modeled as:

$$
\begin{aligned}
\mathbf{H}_{\mathrm{AA}} \mathbf{x}_{\mathbf{A}}(n)-\widehat{\mathbf{H}_{\mathrm{AA}} \mathbf{x}_{\mathbf{A}}}(n) & :=k_{A} \mathbf{H}_{\mathbf{A A}} \mathbf{x}_{\mathbf{A}}(n), \\
\mathbf{H}_{\mathbf{B B}} \mathbf{x}_{\mathbf{B}}(n)-\widehat{\mathbf{H}_{\mathbf{B B}} \mathbf{x}_{\mathbf{B}}}(n) & :=k_{B} \mathbf{H}_{\mathbf{B B}} \mathbf{x}_{\mathbf{B}}(n), \\
\mathbf{H}_{\mathbf{R} \mathbf{R}} \mathbf{x}_{\mathbf{R}}(n)-\widehat{\mathbf{H}_{\mathbf{R} \mathbf{R}} \mathbf{x}_{R}}(n) & :=k_{R} \mathbf{H}_{\mathbf{R} \mathbf{R}} \mathbf{x}_{\mathbf{A}}(n),
\end{aligned}
$$

where $\widehat{\mathbf{H}_{\mathbf{A A}} \mathbf{x}_{\mathbf{A}}}(n), \widehat{\mathbf{H}_{\mathbf{B B}} \mathbf{X}_{\mathbf{B}}}(n)$ and $\widehat{\mathbf{H}_{\mathbf{R} \mathbf{R}} \mathbf{x}_{\mathbf{R}}}(n)$ are the estimations of the self-interference components at terminals $\mathcal{A}$ and $\mathcal{B}$ and relay $\mathcal{R}$, respectively. Typical values for $k_{A}, k_{B}$ and $k_{R}$ have been considered for different types of signal processing canceling techniques in $[33,35]$.

The PLNC concept is specially suitable to enhance the throughput of the TWRC scenarios $[2,19,36]$, where two terminals exchange data with the help of a relay. In the traditional TWRC setup, one terminal would send its message to the relay in the first time slot, the other terminal would use the second time slot for its message and the relay, after applying network coding, would send in the third time slot, the sum of the previously received signals. As the relay only needs the sum of the messages, by using PLNC, the terminals can transmit their signals simultaneously to the relay, in the same time slot, and the relay would then send this sum of the signals in the following time slot, hence, reducing the total required number of communication stages to only two time slots $[19,36]$.

A particular form of PLNC that is based on lattices, and which is dubbed compute-and-forward (CF) $[23,26]$, is implemented in this paper using the algebraic approach proposed in [27]. The main concept is that the relay forwards a function of the superimposed received symbols and that an isomorphism exists between the transmitted codewords and the symbols mapped onto a lattice. The idea relies both on the closeness of group codes under addition and on the additive superposition of electromagnetic waves. Due to these properties, after receiving a combination of the sent codewords and by knowing its own codeword, a terminal may be able to decode the incoming codeword from the other pair. In practice, this isomorphism is framed by using nested lattice codes [26], whose codewords are constructed in the following manner:

$$
\mathcal{L}=\Lambda_{\mathrm{F}} \cap \mathcal{V}_{\Lambda_{\mathrm{C}}}=\left\{\lambda=\left[\lambda_{\mathrm{F}}\right] \bmod _{\Lambda_{\mathrm{C}}}, \lambda_{\mathrm{F}} \in \Lambda_{\mathrm{F}}\right\}
$$

where $\Lambda_{\mathrm{F}}$ is a fine lattice that falls within the fundamental Voronoi region, $\mathcal{V}_{\Lambda_{\mathrm{C}}}$, of a coarse lattice, $\Lambda_{\mathrm{C}}$, and where $\bmod _{\Lambda}$ returns the quantization error with respect to $\Lambda$ [37].

\subsection{Nested lattice code}

Within the algebraic framework of [27], each terminal generates data streams from a two-dimensional integer set with field size $Q=3$, i.e., $S_{A, i}, S_{B, i} \in \mathbb{Z}_{3}^{2}$, for $i=1, \ldots, N_{T}$, which are then mapped onto $\mathbf{x}_{\mathbf{A}}, \mathbf{x}_{\mathbf{B}}, \in \mathbb{C}^{N_{T}}$, with function

$$
\begin{aligned}
\phi: \mathbb{Z}_{3}^{2} \rightarrow \mathcal{L}=\Lambda_{\mathrm{F}} \cap \mathcal{V}_{\Lambda_{\mathrm{C}}}(\in \mathbb{C}) \\
S_{A, i}, S_{B, i} \rightarrow \mathbf{x}_{\mathbf{A}}, \mathbf{x}_{\mathbf{B}}
\end{aligned}
$$

A fine Gaussian lattice is considered, defined as $\Lambda_{\mathrm{F}}=\left\{x \in \mathbb{C}: x=2 z_{1}+j 3 z_{2} ; z=\left(z_{1}, z_{2}\right) \in \mathbb{Z}^{2}\right\}$, and a function $\phi(\cdot)$ is defined such that the transmitted codewords from each antenna of each are mapped onto $\{(0,0),(0,1),(0,2),(1,0),(1,1),(1,2),(2,0),(2,1),(2,2)\} \in$ $\mathbb{Z}_{3}^{2}$ onto the points $\{0+0 j, 0+3 j, 0-3 j, 2+0 j, 2+3 j, 2-$ $3 j,-2+0 j,-2+3 j,-2-3 j\} \in \mathbb{C}$, carving a so-called 9-QAM constellation as the one in [27], defining the nested lattices depicted in Fig. 2. It should be noted that before transmission, the lattice points are normalized to unit power.

\subsection{Protocol for the TWRC}

A major consequence of using massive MIMO is the so-called channel hardening effect [38] resulting from the fact that Gaussian vectors randomly selected in large dimensional space are, with high probability, nearly orthogonal. Due to this effect and by using the mapping $\phi$ defined in Section 3.1, it will be shown below that the task of the relay in the CF protocol becomes the one of finding an integer combination of the transmitted symbols at time slot $n$, in the form:

$$
\left[\mathbf{D}_{\mathbf{A}} \mathbf{x}_{\mathbf{A}}(n)+\mathbf{D}_{\mathbf{B}} \mathbf{x}_{\mathbf{B}}(n)\right] \bmod _{\Lambda_{\mathrm{C}}}
$$

where $\mathbf{D}_{\mathbf{A}}, \mathbf{D}_{\mathbf{B}} \in \mathbb{Z}^{N_{T} \times N_{T}}$ are diagonal matrices with integer entries forming the network code that interprets the effect of a complex channel as an integer one. The relay starts by applying a ZF filter to remove the interference, defined by $\left(\mathbf{H}_{\mathbf{A R}}^{\dagger}+\mathbf{H}_{\mathbf{B R}}{ }^{\dagger}\right)$, where $(\cdot)^{\dagger}$ represents the Moore-Penrose pseudo-inverse. Due to the orthogonality 


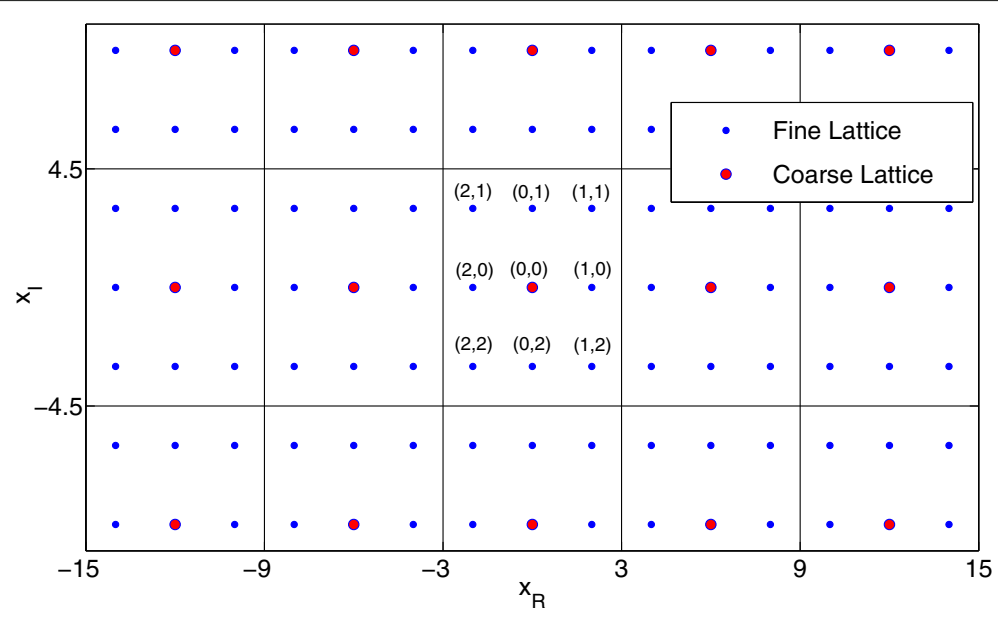

Fig. 2 The used Gaussian nested lattice code with mapping function $\mathbb{Z}_{Q=3}^{2} \rightarrow \mathbb{C}: x=\phi(S)$

induced by the massive array, this is in fact a quasi-optimal approach [17]. Therefore, the pseudo-inverses of $\mathbf{H}_{\mathrm{AR}}$ and $\mathbf{H}_{\mathbf{B R}}$ are calculated, and the received vector at the relay is given by:

$$
\begin{aligned}
\mathbf{y}_{\mathbf{P}}(n)= & \mathbf{H}_{\mathbf{A R}}{ }^{\dagger} \mathbf{y}_{\mathbf{R}}(n)+\mathbf{H}_{\mathbf{B R}}{ }^{\dagger} \mathbf{y}_{\mathbf{R}}(n) \\
= & \left(\mathbf{H}_{\mathbf{A R}}{ }^{\dagger} \mathbf{H}_{\mathbf{A R}} \mathbf{x}_{\mathbf{A}}(n)+\mathbf{H}_{\mathbf{B R}}{ }^{\dagger} \mathbf{H}_{\mathbf{B R}} \mathbf{x}_{\mathbf{B}}(n)\right)+ \\
& \left(\mathbf{H}_{\mathbf{B R}}{ }^{\dagger} \mathbf{H}_{\mathbf{A R}} \mathbf{x}_{\mathbf{A}}(n)+\mathbf{H}_{\mathbf{A R}}{ }^{\dagger} \mathbf{H}_{\mathbf{B R}} \mathbf{x}_{\mathbf{B}}(n)\right)+ \\
& \left(\mathbf{H}_{\mathbf{A R}}{ }^{\dagger}+\mathbf{H}_{\mathbf{B R}}{ }^{\dagger}\right)\left(k_{R} \mathbf{H}_{\mathbf{R} \mathbf{R}} \mathbf{x}_{\mathbf{R}}(n)+\mathbf{n}_{\mathbf{R}}(n)\right) \\
= & \underbrace{\mathbf{D}_{\mathbf{A}} \mathbf{x}_{\mathbf{A}}(n)+\mathbf{D}_{\mathbf{B}} \mathbf{x}_{\mathbf{B}}(n)}_{\text {desired component }}+\underbrace{\tilde{\mathbf{n}_{\mathbf{R}}}(n)}_{\text {equivalent total noise }},
\end{aligned}
$$

where $\mathbf{y}_{\mathbf{P}}(n) \in \mathbb{C}^{N_{T} \times 1}$ is the desired linear combination of the terminals' signals that arrive at the relay. Additionally, without loss of generality, one considers the case where the transmit power of both terminals and the one of the relay are $p_{A}=p_{B}=p_{R}=1$. It is interesting to look at the equivalent noise in (7) given the noise enhancement caused by ZF. The simplifications are a consequence of the channel hardening effect, which ensures all the following asymptotic properties for the matrices: $\mathbf{H}_{\mathrm{AR}}{ }^{\dagger} \mathbf{H}_{\mathbf{B R}} \rightarrow$ $\mathbf{0}, \mathbf{H}_{\mathbf{B R}}{ }^{\dagger} \mathbf{H}_{\mathrm{AR}} \rightarrow \mathbf{0}$, as $M_{R} \rightarrow \infty$. Furthermore, the selfinterference is also mitigated due to the orthogonality between $\left(\mathbf{H}_{\mathbf{A R}}+\mathbf{H}_{\mathbf{B R}}\right)^{\dagger}$ and $\mathbf{H}_{\mathbf{R R}}$, while $\mathrm{ZF}$ detection in massive MIMO does not lead to a large noise enhancement, in contrast to what happens in conventional MIMO. The proposed CF protocol for IBFD relaying with massive MIMO is detailed in Algorithm 1, describing the processing that is performed both at the relay and at the two terminals. It should be noted again that when the relay transmits, it only uses $M_{T}=N_{T}$ antennas, such that $\mathbf{H}_{\mathbf{R A}}$ is symmetric and full-rank with high probability. The performance of this scheme will be assessed in Section 5 .

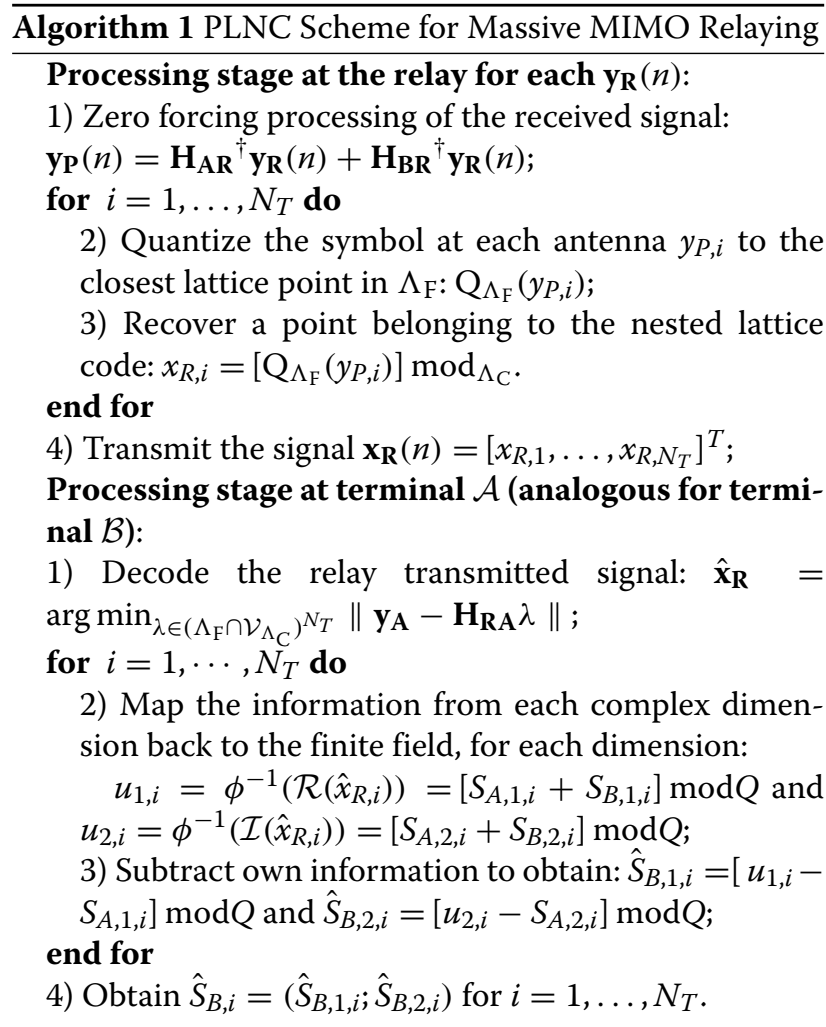

\section{The three-users case: the Y-network}

Let us now consider a relay serving three users. In this setup, having a conventional MIMO relay suffices in order to have the three terminals exchanging the messages between them using only one time slot per message exchange on average. One should however note that, as typical with IBFD, the number of antennas at the relay with IBFD is the double of the ones in a half-duplex system. 


\subsection{Configurations for message exchanging}

The Y-network adopted in this work is depicted in Fig. 3, depicting the messages that are exchanged in the case of a TDMA operation mode. In that case, the relay needs to receive three messages, each of which coming from a different terminal (dashed red lines), and it later needs to broadcast each of them, so that each user gets the two messages that it still does not know from the other two users (solid blue lines), amounting to a total of six time slots for the message exchanging process to take place.

When using the multiple-access channel (MAC) phase of the schemes proposed in [11], one creates a virtualMIMO uplink with three streams (one from each terminal to the relay), where the relay receives the incoming symbols from the three terminals using three antennas. In the proposed setups, both the relay and the terminals have extra antennas to support IBFD: the relay is equipped with three receiving antennas and three transmitting antennas, using the so-called natural isolation between transmit and receive antennas, using radio frequency $(\mathrm{RF})$ cancellation, and finally counting on signal processing to assure the remaining signal cancellation [5, 35, 39, 40]. On the other side, each terminal has one transmitting antenna and either one or two receiving antennas (for MIMO reception in the latter case). In fact, two different configurations for the terminals' side will be studied, while at the relay three receiving antennas and three transmitting antennas are considered in both configurations. The first configuration, denoted hereafter as configuration A, considers terminals with one receiving antenna and one transmitting antenna, so as to enable IBFD (Fig. 5). The

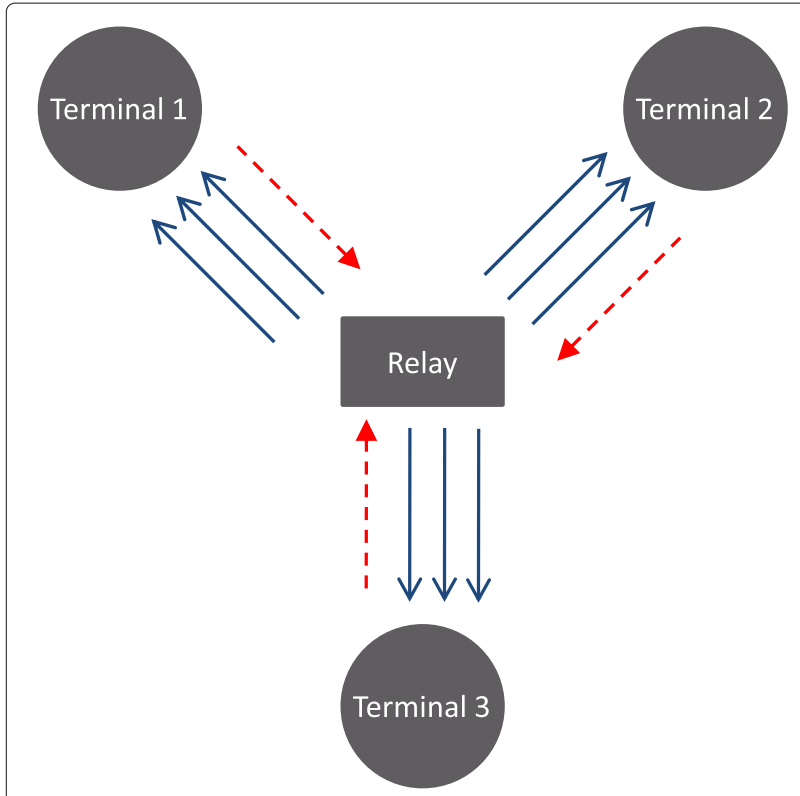

Fig. 3 The Y-network configuration with three terminals and one relay second configuration, denoted as configuration B, comprises the same number of antennas at the relay, but each terminal has now two receiving antennas (Fig. 6), enabling MIMO detection in the downlink (from the relay to each terminal).

Figure 4 illustrates the transmission phase from the IBFD terminals to the relay. Figures 5 and 6 show the broadcast phase, when the relay transmits to the terminals while suppressing LI at the relay; Fig. 5 shows the setup with two receive antennas at the terminals, and Fig. 6 shows the setup with three receive antennas. In all these three figures, the LI is represented by the dotted lines, which sets this model apart from the one in [11].

Before looking into the details of the system model, as it will be detailed in Section 4.2, one starts by looking at the overall process of exchanging messages when employing IBFD with configuration B, as depicted in Fig. 7. In this configuration, the receiver is able to detect the two unknown incoming messages, given that it already knows its own message and can cancel it out using the PLNC principle. The arrows in Fig. 7 are associated to the messages exchanged along the successive time slots, where $x_{i, j}$ represents a message sent from terminal $i$ during time slot $j$. In the first time slot, the relay does not have anything to send to the terminal, and for that reason, it remains silent. After this initial stage, the transmit antennas of the three terminals and the ones of the relay are all sending data streams, resulting on an average of one time slot per information exchange. It should be noted the delay of one time slot in the downlink regarding the information that is sent in the uplink.

\subsection{PHY signaling and detection}

For a given time slot $n>1$, the signal received at the relay is

$$
\mathbf{y}(n)=\mathbf{H}(n) \mathbf{x}(n)+\mathbf{n}(n)+\mathbf{H}_{L I}(n) \hat{\mathbf{x}}(n-1),
$$

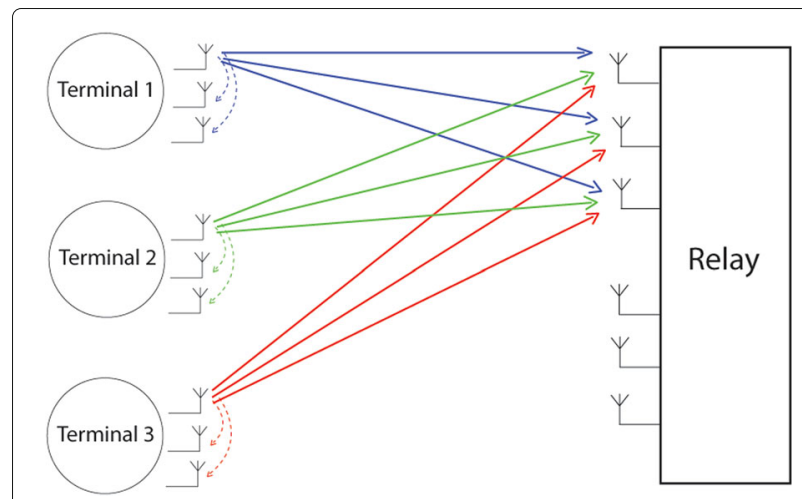

Fig. 4 Uplink phase with "full-duplex" (the third antenna of each terminal is not used in configuration A—cf. Fig. 5) 


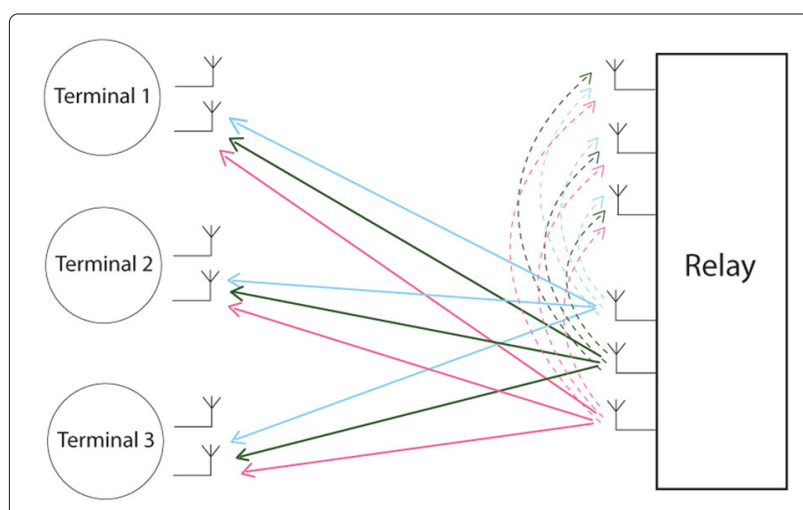

Fig. 5 Downlink phase with "full-duplex" (configuration A)

where $\mathbf{y}(n)=\left[y_{1}(n), y_{2}(n), y_{3}(n)\right]^{\top}$ is the received signal vector, $\mathbf{x}_{n}=\left[x_{1}(n), x_{2}(n), x_{3}(n)\right]^{\top}$ denotes the transmitted signal vector, $\mathbf{H}(n)$ stands for the channel matrix where each entry $h_{i, k}(n)$ represents, as before, the link between the transmitting antenna of the $k$ th terminal and the $i$ th receiving antenna of the relay, $\mathbf{n}(n)$ corresponds to the noise vector, $\hat{\mathbf{x}}(n-1)=\left[\hat{x}_{1}(n), \hat{x}_{2}(n), \hat{x}_{3}(n)\right]^{\top}$ denotes the previously detected symbol at the relay and $\mathbf{H}_{L I}(n)$ stands for the channel matrix of the LI at the relay. On the other hand, the signal that each terminal receives in the same time $\operatorname{slot} n$ is given by

$$
\mathbf{y}^{\prime}(n)=\mathbf{H}(n) \hat{\mathbf{x}}(n-1)+\mathbf{n}^{\prime}(n)+\mathbf{H}_{L I}^{\prime}(n) \mathbf{x}(n)
$$

where $\mathbf{y}^{\prime}(n)$ represents the received signal vector with $N_{T}$ complex dimensions (i.e., $N_{T}$ antennas), $\mathbf{n}^{\prime}(n)$ corresponds to the noise vector and $\mathbf{H}_{L I}^{\prime}(n)$ denotes the channel matrix of the LI at the terminals. Note that (9) still holds for the case with $N_{T}=1$ in configuration A, although in that case all vectors become scalars.

The LI contribution in both (8) and (9) cannot be neglected and leads to a performance loss; hence, some type of isolation (physical and electrical) must be added between the corresponding pair transmitting/receiving

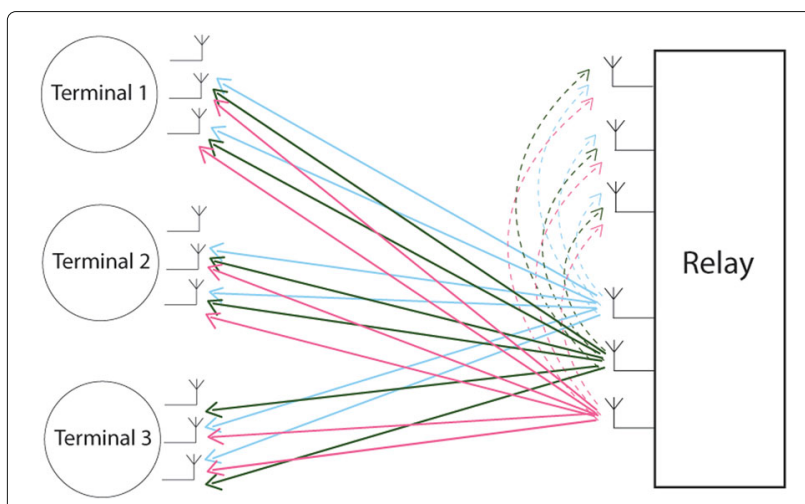

Fig. 6 Downlink phase with "full-duplex" (configuration B) antenna. Similarly, to the TWRC model in (4), the residual LI will be represented by a $K$ factor, with $K<1$, as considered in [33], i.e., a lower $K$ value represents a larger reduction of the self-interference, yielding the following updated expressions:

$$
\begin{aligned}
\mathbf{y}(n) & =\mathbf{H}(n) \mathbf{x}(n)+\mathbf{n}(n)+K \mathbf{H}_{L I}(n) \hat{\mathbf{x}}(n-1) \\
\mathbf{y}^{\prime}(n) & =\mathbf{H}(n) \hat{\mathbf{x}}(n-1)+\mathbf{n}^{\prime}(n)+K \mathbf{H}_{L I}^{\prime}(n) \mathbf{x}(n) .
\end{aligned}
$$

Each element of the different channel matrices is taken from a zero-mean circularly symmetric complex Gaussian distribution with unit variance, and the noise components are drawn from an independent circularly symmetric complex Gaussian with zero average and variance $\sigma_{n}^{2}$. It is also assumed that the channel state information at the receiver (CSIR) is available and that, as reflected in (10) and (11), all the links between terminals and the relay are reciprocal, i.e., they are the same in the uplink and downlink phases (when this assumption is verified in real systems, it simplifies the channel estimation phase). Standard $M$-ary squared quadrature amplitude modulation (M-QAM) constellations are used to transmit the different messages. The symbols are taken from a finite complex constellation $\mathcal{C}$ constructed from the Cartesian product $\mathcal{C}=\mathcal{C}_{R} \times \mathcal{C}_{R}$, where $\mathcal{C}_{R}$ is the real set

$\mathcal{C}_{R}=\{-(\sqrt{M}-1), \ldots,-3,-1,+1,+3, \ldots,+(\sqrt{M}-1)\}$.

Without loss of generality, the filters adopted for the performance assessment at the receivers have a normalized impulse response $h(t)$ such that $\int|h(t)|^{2} d t=1$.

Finally, the symbol error rate (SER) of the downlink phase is obtained by comparing the messages decoded at each terminal or relay with the original messages sent by each of them in the uplink phase. For the case of the relay, the signal-to-noise ratio (SNR) is defined by

$\mathrm{SNR}=$

$\frac{E\left\{\mathbf{y}(n)^{\top} \mathbf{y}(n)\right\}}{E\left\{\mathbf{n}(n)^{\top} \mathbf{n}(n)\right\}+K^{2} E\left\{\hat{\mathbf{x}}(n-1)^{\top} \mathbf{H}(n-1)^{\top} \mathbf{H}_{L I}(n) \hat{\mathbf{x}}(n-1)\right\}}$,

as in similar IBFD systems [33]. Likewise, a similar expression can be written for the terminals' side. Note that the downlink performance accumulates the errors occurred during the two phases, i.e.,

$$
\mathrm{SER}_{\text {total }}=1-(1-\mathrm{SER})^{2} .
$$

\subsection{Protocols for the Y-network}

The IBFD strategies proposed in this paper evolve from the ones presented in [11], with the uplink and downlink phases now being merged in the same time slot, allowing to double the overall throughput. 


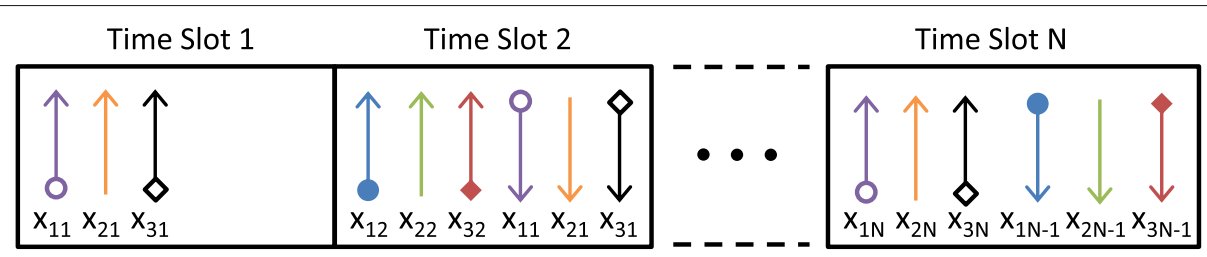

Fig. 7 Messages flow between the terminals and the relay using configuration $B$ (terminals with two receiving antennas)

In the uplink phase, the same strategy is used for both configuration A and configuration B, using virtual MIMO: the signals are transmitted simultaneously by the three terminals, and the relay applies a robust detection technique such as a lattice reduction-aided (LRA) detector, followed by ordered successive interference cancellation with minimum mean square error (OSIC-MMSE) [41]. It is well known in the MIMO literature that the performance attained with LRA captures the full diversity order available in the MIMO spatial multiplexing, i.e., the slope of the SER curves is the same as the one provided by the ML detection, although they exhibit some power penalty in respect to the ML performance curves. The option for this type of MIMO detection algorithm is due to the fact that lattice-based receivers are until now the best compromise between computational complexity and performance [41]. At the end of this MIMO detection phase, the relay has detected the messages $x_{1}, x_{2}$ and $x_{3}$; this procedure consumes one time slot in the overall messages exchanging process.

The downlink phase in configuration A consists of the following three steps during the broadcast phase:

Step 1: The relay first broadcasts the estimates of the signal received during the previous time slot (i.e., during the MAC phase).

Step 2: Using the PLNC principle, each terminal receives the above overlapped messages and cancels its own additive contribution (given that each terminal knows the signal it has previously transmitted, as well as the channel response, assuming CSIR).

Step 3: Each terminal estimates the two remaining messages of the other two terminals performing a joint ML detection for those two remaining symbols.

During the downlink phase of configuration $B$, the first cancellation described for configuration A (in step 2) is performed in the same manner at all the three terminals; however, since two antennas are used for reception in configuration $\mathrm{B}$, the remaining detection problem can be seen as a $2 \times 2$ MIMO spatial multiplexing problem, which can be dealt with by one of the many different detection techniques, according to the complexity-performance tradeoff one needs and is able to afford [41]. For this purpose, LRA OSIC-MMSE was chosen to obtain the results, i.e., the same detection algorithm as the one employed at the relay in the first phase. Note that the downlink phase consumes one time slot, independently of the configuration used at the terminals.

One can now consider the time evolution of this proposed scheme for the Y-network, further looking at Fig. 7. Consider that five messages from each terminal are to be exchanged with the other terminals: the first time slot is solely used for the first MAC phase (i.e., the uplink of the messages to the relay); the second time slot is used for the first downlink phase of data, and simultaneously for the second uplink phase (i.e., for the second messages of the terminals); this procedure is repeated until the sixth time slot, which is only used for the last downlink phase of information. Hence, six time slots are required for all the messages to be exchanged between the terminals. In general, the downlink phase corresponding to the $(n-1)$ th message uploaded to the relay is performed during the same time slot as the uplink phase of the next $n$th message; consequently, the number of slots required to exchange $N$ messages is $N+1$ and so, when $N$ becomes large, the scheme accomplishes the message exchange between all terminals in the Y-network using only one time slot on average.

\section{Performance results}

\subsection{The two-users case: the TWRC}

The performance of the proposed protocol for the TWRC is numerically evaluated in terms of the SER performance for $M_{T}=N_{T}=2$ antennas (as in Fig. 1), then the effect of of $M_{R}$ on SER is studied, and finally, one assesses the robustness of the system to the existence of estimation errors in all the channel matrices involved. All results are obtained via Monte Carlo simulation, using uncoded MIMO.

\subsubsection{Impact of different $M_{R}$ antennas at the receiver}

The orthogonalisation of the MIMO channel occurs as the number of antennas at the relay tends to infinity. The results in Fig. 8 evaluate the effect of having a finite number of receive antennas at the relay. The simulation assumes that each channel has entries taken from 


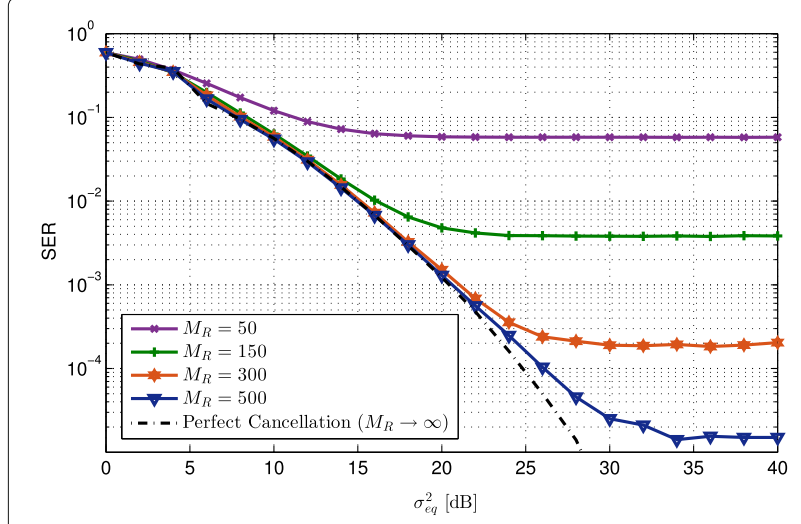

Fig. 8 SER performance of the bidirectional CF massive MIMO protocol for different numbers of relay receiving antennas $M_{R}$ and different equivalent noise power levels $\sigma_{e q}^{2}$

a normalized complex Gaussian distribution $\mathcal{C N}(0,1)$. The SER evolution is plotted against the equivalent noise power, which considers a fixed self-interference mitigation gain, that is equal for the three system elements, being $k=k_{A}=k_{B}=k_{R}$, and a varying thermal noise $\sigma^{2}=\sigma_{\mathbf{n}_{\mathbf{A}}}^{2}=\sigma_{\mathbf{n}_{\mathbf{B}}}^{2}=\sigma_{\mathbf{n}_{\mathbf{R}}}^{2}$, such that the equivalente noise is $\sigma_{e q}^{2}=k+\sigma^{2}$.

The asymptotic effect is clear in Fig. 8, where, for a low number of antennas, the orthogonal properties of large dimension arrays do not hold. For the different $M_{R}$ antennas considered at the relay, the SER curves stall at an error floor (caused by the LI) that decreases with $M_{R}$ and is caused by the interference components that are not properly canceled due to the reminiscent orthogonality-defect.

When considering a very larger number of antennas, for example $M_{R}=500$, the effect of imperfect cancellation of the leak between the MIMO spacial channels (i.e., when a perfect orthogonalisation of the channel is not achieved) tends to be negligible, as the orthogonal property is valid for a large range of $\sigma_{e q}^{2}$, up to close to $25 \mathrm{~dB}$. Moreover, the noise floors appear at acceptable values of SER, and when $M_{R}=\infty$, the SER tends to the asymptotic case of perfect interference cancellation. This allows to have a PHY layer that is fourfold more efficient in terms of throughput, while providing a service quality to the upper layers that is similar to the one with the traditional TDMA approach. In fact, considering a typical target SER for wireless channels around $10^{-3}$, this is clearly achieved when observing Fig. 8, even for $M_{R}=300$ antennas.

\subsubsection{Impact of imperfect channel estimation}

Another interesting aspect is to evaluate how imperfect CSI may deteriorate the performance. To that end, consider that the relay only has access to erroneous estimations of the channel matrices, i.e., each entry of the channel matrices is known at the relay apart from some error component. Thus, we assume for all channel matrices that $\mathbf{H}=\tilde{\mathbf{H}}+\mathcal{E}_{\mathbf{H}}$, where the error component is generated from a complex Gaussian distribution $\mathcal{C N}\left(0, \sigma_{\mathbf{H}}^{2}\right)$, with the variance $\sigma_{\mathbf{H}}^{2}$ accounting for the power of the estimation error. Figure 9 depicts the average SER performance for different values of the equivalent noise, different numbers of antennas and different estimation error power.

Imperfect estimation of the channel matrices is still a major drawback in the proposed CF protocol with massive MIMO. For $M_{R}=150$ antennas (blue curves in Fig. 9), when the relay does not exactly know the channel matrices, the SER curves for $\sigma_{\mathbf{H}}^{2}=10^{-5}$ and for $\sigma_{\mathbf{H}}^{2}=10^{-3}$ lead to an error floor. This is caused not only by the noise enhancement caused by ZF filtering but also by the fact

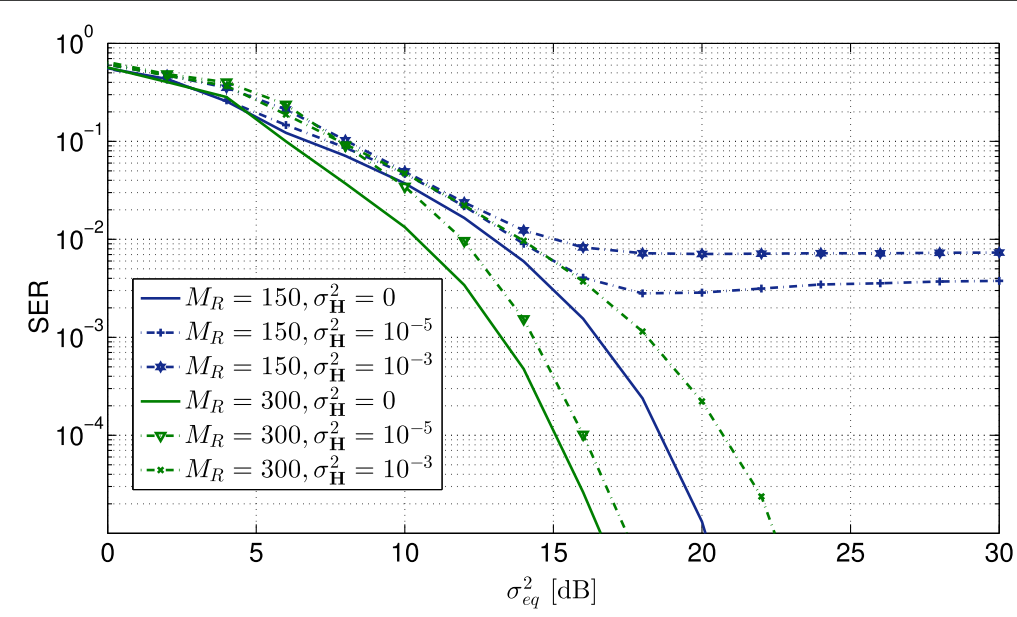

Fig. 9 SER curves of the CF massive MIMO protocol for different numbers of relay receiving antennas $M_{R}$, different interference power levels $\sigma_{\text {eq }}^{2}$ and different channel estimation errors power $\sigma_{\mathbf{H}}^{2}$ 
that $\mathbf{D}_{\mathbf{A}}$ and $\mathbf{D}_{\mathbf{B}}$ will no longer be diagonal (i.e., a unitary network code is never achieved) changing the geometry of the lattice to a non-orthogonal one, which cannot be handled by the CF scheme.

When a larger number of antennas is considered, as the green curves in Fig. 9 show for $M_{R}=300$, and for the same power of the channel estimation error, the error floor disappears (for the depicted SER values) due to the channel hardening effect of large dimensional Gaussian matrices. Nevertheless, these SER curves will eventually stall at an error floor for lower values of SER.

One should note that when increasing the number of antennas $M_{R}$ to a few hundreds antennas, the SER floor decreases to the desired typical values in wireless links $\left(\approx 10^{-3}\right)$, before error correction takes place.

\subsection{The three-users case: the $Y$-network}

The two configurations evaluated in the paper for the Y-network were always simulated with LRA with OSICMMSE detection at the relay and also at the terminals (in the MIMO detection stage that exists in configuration B). Alternatively, joint ML detection (i.e., "brute force") is adopted in configuration A when estimating the two final remaining messages. Figures 10 and 11 respectively show the performance results in terms of the overall SER for 4QAM and 16-QAM, using the same constellations in both the uplink and downlink phases, and using the same interference factors $K$ used in [33]: $K=10^{-3}, 10^{-2}, 0.5 \times 10^{-2}$ and $10^{-1}$. Note that both schemes achieve the goal of spending just one time slot per each messages exchange between terminals (with 16-QAM allowing for a more spectrally efficient system). In Fig. 11, one can observe that configuration $\mathrm{A}$, in which a joint $\mathrm{ML}$ detection of two (remaining) different messages takes place with only one antenna, exhibits a rather poor performance when using 16-QAM. This is a consequence of a very small Euclidean distance between the symbols resulting from the two received remaining messages after the terminal suppresses its own message; for example, in the case of the detection at terminal 1 , the joint ML decision is applied to the sum $h_{2} x_{2}+h_{3} x_{3}$. Hence, configuration A can only be used for binary or quaternary modulations.

The results in Figs. 10 and 11 quantify how much isolation for IBFD communication is required in order to attain a certain targeted performance. As expected, from (10), one can observe in both figures that for a higher interference factor $K$, the performance deteriorates in all cases. In Fig. 10, one can see that for SNR $=35 \mathrm{~dB}$ configuration $B$ attains SER $=10^{-3}$, a typical objective for uncoded wireless transmission. Even in the case of the simpler configuration $\mathrm{A}$, an $\mathrm{SNR}>30 \mathrm{~dB}$ guarantees a SER below $10^{-2}$. Figure 10 shows that the performance is quite similar for $K=10^{-2}$ or $K=10^{-3}$; in other words, for applications that do not usually operate at high SNR,

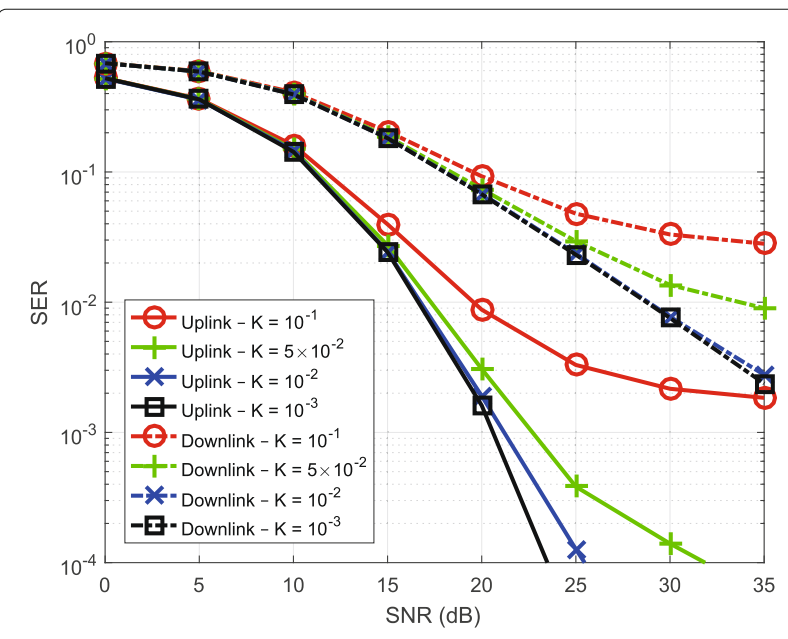

(a)

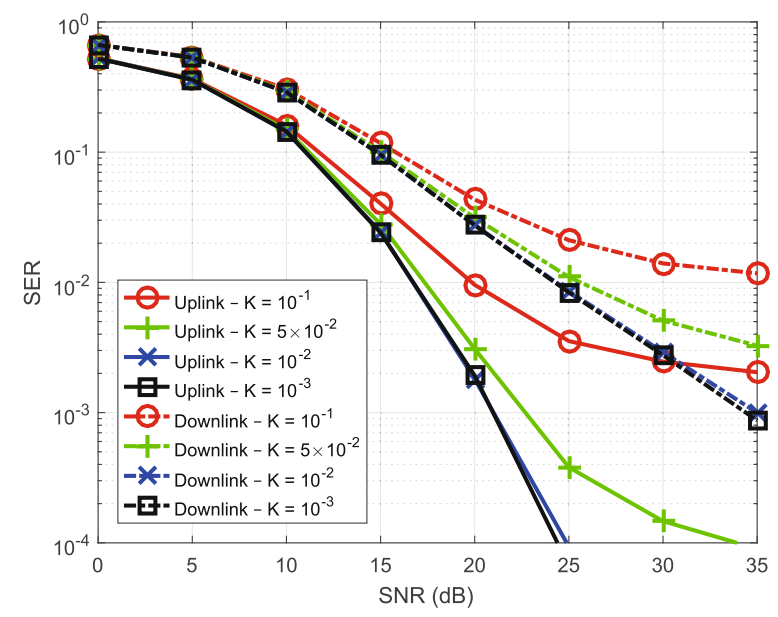

(b)

Fig. 10 Performances of the two configurations in the Y-network with 4-QAM in both phases. Symbol error rates at the relay (uplink) and at each of the terminals (uplink followed by downlink). a Configuration A. b Configuration B

one only needs to optimize the LI isolation up to a certain point, allowing a simpler and cheaper concatenation of isolation and LI cancellation methods. Interestingly, the performance in the low SNR regime does not depend much on the interference term, because thermal noise is the dominant term in that regime.

Given that in configuration B the detection at the terminals of the last two remaining symbols is undertaken with MIMO spacial diversity of order two [41], this configuration surpasses the performance of the construction A counterpart when having the same $K$ factor and the same modulation. This is observed in the figures for configuration B in the downlink phase (the solid lines in all figures) by noticing the doubling of the slope of the curves. Nonetheless, this gain comes at the expense of the complexity involved in LRA OSIC-MMSE detection [41]. 


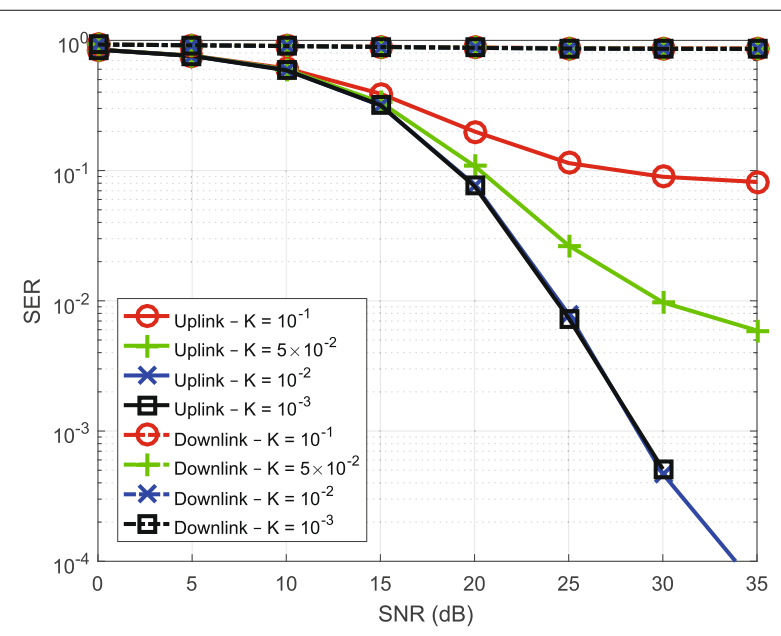

(a)

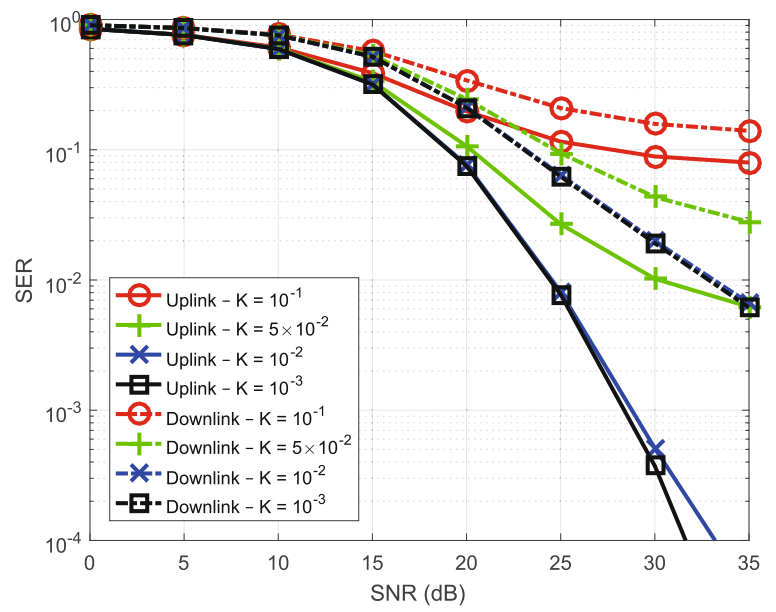

(b)

Fig. 11 Performances of the two configurations in the Y-network with 16-QAM in both phases. Symbol error rates at the relay (uplink) and at each of the terminals (uplink followed by downlink). a Configuration A. b Configuration B

Note that the performance in the uplink phase is the same for both configurations, given that the MAC phase involves the same virtual-MIMO channel in both configurations. Another observation in both Figs. 10 and 11 is that the performance of the downlink phase is worse than the uplink phase, due to the fact that the former bears the cumulative errors that occur in both the uplink (MAC) and the downlink (broadcast) phases-cf. (14).

\section{Conclusions}

The paper argues that the immense progress that the physical layer of wireless communications has faced in the last decade offers fruitful opportunities to redesign the protocols in the layers above. This paper has shown that the coexistence of MIMO and massive MIMO, IBFD, and
PLNC allows to greatly reduce the number of orthogonal channels that are needed to exchange messages in relaycentered networks, which translate to the use of many fewer time slots to accomplish message exchanges. Both in the case of the TWRC and the case of the Y-network, it is feasible to asymptotically exchange information between all users using only one time slot on average, instead of the traditional four and six time slots spent in the traditional TDMA approach, respectively, for the TWRC and the Y-network.

In the case of the TWRC, the benefits of having a massive MIMO array allows to use a simple latticebased PLNC to establish the bidirectional information flow. Massive MIMO not only plays a central role in reducing the inherent interference between the two data flows but also helps overcoming the self-interference at the relay when the number of receiving antennas at the relay increases to a few hundreds. The latter effect was observed via SER curves using typical power levels for the residual self-interference that appear when using stateof-the-art cancellation techniques. Finally, the impact of imperfect CSI (both of the primary links and the loopback interference links) has also been analyzed. The system's performance is shown to be chiefly dependent on the number of antennas at the relay and also on the channel state information of all the channels involved. For relays with a few hundred antennas, the proposed scheme with only one time slot per message exchange is feasible.

In the case of the Y-network, by assessing the performance of the proposed setups for typical levels of residual interference, it was shown that, for good levels of interference cancellation, interference can in fact be well tolerated while allowing doubling the time efficiently of the message exchange mechanism, even when using conventional MIMO in both terminals and also at the relay, while running standard detection algorithms such as LRA detection. For typical values of LI, a sufficient uncoded performance is still achievable at the terminals. In fact, for the more demanding 16-QAM modulation, having two receive antennas at the terminals is mandatory, such that the MIMO detection diversity of order two allows to separate the two remaining data streams after the initial phase when the terminal cancels its own data.

One should note that the complexity involved in putting together the three techniques is no more than the sum of the individual complexity associated to each. One further improvement one can add to the physical layer is the optimization of the powers at the relay and also at the terminals in order to maximize the sum-rate of the system. However, that leads to non-trivial optimization problems that potentially will have to be solved under strict time constraints dictated by the time correlation of the channels. A more straightforward extension to this work is 
the assessment of OFDM, given that this is a modulation designed to cope with wide-band frequency selective channels and used in the recent versions of the 802.11 family of standards, as well as in $4 \mathrm{G}$ celular systems. Another very recent technology that is presently at an early stage of research is power-domain non-orthogonal multiple access (NOMA) [42-45], and the exploration of NOMA with MIMO-IBFD is a major research problem that needs to be addressed. Moreover, as PLNC can be seen as akin to the NOMA concept, a fruitful interplay between these two techniques can be expected.

\section{Acknowledgements}

This work was funded by FCT (Foundation for Science and Technology) under project UID/EEA/50008/2013. Francisco A. Monteiro is also grateful to the European COST Action IC1 104 - "Random Network Coding and Designs over $\mathrm{GF}(\mathrm{q})^{\prime \prime}$ for having provided funding for learning opportunities in the field of network coding

\section{Competing interests}

The authors declare that they have no competing interests.

\section{Author details}

${ }^{1}$ Instituto de Telecomunicações, Lisbon, Portugal. ${ }^{2}$ Instituto Superior Técnico, Universidade de Lisboa, Lisbon, Portugal. ${ }^{3}$ ISCTE_-Instituto Universitário de Lisboa, Lisbon, Portugal.

\section{Received: 17 August 2016 Accepted: 24 February 2017}

Published online: 22 May 2017

\section{References}

1. G Zheng, I Krikidis, C Masouros, S Timotheou, DA Toumpakaris, Z Ding, Rethinking the role of interference in wireless networks. IEEE Commun. Mag. 52(11), 152-158 (2014)

2. G Liu, FR Yu, H Ji, VCM Leung, X Li, In-band "full-duplex" relaying: a survey, research issues and challenges. IEEE Commun. Surv. Tutor. 17(2), 500-524 (2015)

3. D Kim, H Lee, D Hong, A survey of in-band "full-duplex" transmission: from the perspective of PHY and MAC layers. IEEE Commun. Surv. Tutor. 17(4), 2017-2046 (2015)

4. HQ Ngo, HA Suraweera, M Matthaiou, EG Larsson, Multipair "full-duplex" relaying with massive arrays and linear processing. IEEE J. Sel. Areas Commun. 32(9), 1721-1737 (2014)

5. JS Lemos, FA Monteiro, I Sousa, A Rodrigues, in 2015 IEEE Global Conference on Signal and Information Processing (GlobalSIP). Full-duplex relaying in MIMO-OFDM frequency-selective channels with optimal adaptive filtering (Orlando, 2015), pp. 1081-1085

6. F Boccardi, RW Heath, A Lozano, TL Marzetta, P Popovski, Five disruptive technology directions for 5G. IEEE Commun. Mag. 52(5), 74-80 (2014)

7. B Roman, I Wassell, I Chatzigeorgiou, Scalable cross-layer wireless access control using multi-carrier burst contention. IEEE J. Sel. Areas Commun. 29, 113-128 (2011)

8. RH Louie, Y Li, B Vucetic, Practical physical layer network coding for two-way relay channels: performance analysis and comparison. IEEE Trans. Wirel. Commun. 9(2), 764-777 (2010)

9. N Lee, JB Lim, J Chun, Degrees of freedom of the MIMO Y channel: signal space alignment for network coding. IEEE Trans. Inf. Theory. 56(7), 3332-3342 (2010)

10. A Chaaban, K Ochs, A Sezgin, in 2013 IEEE International Symposium on Information Theory. The degrees of freedom of the MIMO Y-channel (Istanbul, 2013), pp. 1581-1585

11. FS Brás, FE Ferreira, FA Monteiro, A Rodrigues, in 2014 IEEE Workshop on Signal Processing Systems (SiPS). Interference suppression with physical-layer network coding and MIMO for multi-way channels (Belfast, 2014), pp. 1-6

12. FE Ferreira, FA Monteiro, I Sousa, in 2016 18th Mediterranean Electrotechnical Conference (MELECON). Full-duplex MIMO and PLNC for the Y-network (Limassol, 2016), pp. 1-5
13. M Duarte, A Sabharwal, in Conference Record of the 44th Asilomar Conference on Signals, Systems and Computers (ASILOMAR). Full-Duplex Wireless Communications Using Off-the-Shelf Radios: Feasibility and First Results (Pacific Grove, 2010), pp. 1558-1562

14. S Hong, J Brand, JI Choi, M Jain, J Mehlman, S Katti, P Levis, Applications of self-interference cancelation in $5 \mathrm{G}$ and beyond. IEEE Commun. Mag. 52(2), 114-121 (2014)

15. D Bharadia, McE Milin, S Katti, in Proceedings of the 2013 ACM SIGCOMM conference. SIGCOMM '13. Full Duplex Radios (Hong Kong, 2013), pp. 375-386

16. M Jain, Jl Choi, T Kim, D Bharadia, S Seth, K Srinivasan, P Levis, S Katti, P Sinha, in Proceedings of the 17th Annual International Conference on Mobile Computing and Networking. Practical, Real-time, Full Duplex Wireless MobiCom '11 (Las Vegas, 2011), pp. 301-312

17. F Rusek, D Persson, BK Lau, EG Larsson, TL Marzetta, O Edfors, F Tufvesson, Scaling up MIMO: opportunities and challenges with very large arrays. IEEE Signal Process. Mag. 30, 40-60 (2013)

18. B Nazer, M Gastpar, Reliable physical layer network coding. Proc. IEEE. 99(3), 438-460 (2011)

19. P Popovski, H Yomo, in 2007 IEEE International Conference on Communications. Physical Network Coding in Two-Way Wireless Relay Channels (Glasgow, 2007), pp. 707-712

20. R Ahlswede, N Cai, SY Li, R Yeung, Network Information Flow. IEEE Trans. Inf. Theory. 46(4), 1204-1216 (2000)

21. S Zhang, SC Liew, PP Lam, in Proceedings of the 12th Annual International Conference on Mobile Computing and Networking. Hot Topic: Physical-Layer Network Coding, MobiCom '06 (Los Angeles, 2006), pp. 358-365

22. P Popovski, $\mathrm{H}$ Yomo, in IEEE International Conference on Communications (ICC). The Anti-Packets Can Increase the Achievable Throughput of a Wireless Multi-Hop Network, Volume 9 (Istanbul, 2006), pp. 3885-3890

23. B Nazer, M Gastpar, in IEEE International Symposium on Information Theory (ISIT). Computing over Multiple-Access Channels with Connections to Wireless Network Coding (Seattle, 2006), pp. 1354-1358

24. R Chang, SJ Lin, WH Chung, Symbol and bit mapping optimization for physical-layer network coding with pulse amplitude modulation. IEEE Trans. Wirel. Commun. 12(8), 3956-3967 (2013)

25. M Wilson, K Narayanan, H Pfister, A Sprintson, Joint physical layer coding and network coding for bidirectional relaying. IEEE Trans. Inf. Theory. 56(11), 5641-5654 (2010)

26. B Nazer, M Gastpar, Compute-and-forward: harnessing interference through structured codes. IEEE Trans. Inf. Theory. 57(10), 6463-6486 (2011)

27. C Feng, D Silva, FR Kschischang, An algebraic approach to physical-layer network coding. IEEE Trans. Inf. Theory. 59(11), 7576-7596 (2013)

28. B Yin, M Wu, C Studer, J Cavallaro, J Lilleberg, in Conference Record of the 47th Asilomar Conference on Signals, Systems and Computers (ASILOMAR). Full-Duplex in Large-Scale Wireless Systems (Pacific Grove, 2013), pp. 1623-1627

29. T Riihonen, Vehkapera M, R Wichman, in 47th Annual Conference on Information Sciences and Systems (CISS). Large-System Analysis of Rate Regions in Bidirectional Full-Fuplex MIMO Link: Suppression versus Cancellation (Baltimore, 2013), pp. 1-6

30. TRiihonen, S Werner, R Wichman, in Conference Record of the 43th Asilomar Conference on Signals, Systems and Computers (ASILOMAR). Spatial Loop Interference Suppression in Full-Duplex MIMO Relays (Pacific Grove, 2009), pp. 1508-1512

31. W Zhang, X Ma, B Gestner, DV Anderson, in 45th Annual Conference on Information Sciences and Systems (CISS). Designing Low-Complexity Equalizers for Wireless Systems (Baltimore, 2011), pp. 1-6

32. G Zheng, Joint beamforming optimization and power control for "full-duplex" MIMO two-way relay channel. IEEE Trans. Signal Process. 63(3), 555-566 (2015)

33. STedik, GK Kurt, in 2014 IEEE 79th Vehicular Technology Conference (VTC Spring). Practical Full Duplex Physical Layer Network Coding (Seoul, 2014), pp. 1-4

34. Y Sun, Y Yang, P Si, R Yang, Y Zhang, Novel self-interference suppression schemes based on Dempster-Shafer theory with network coding in two-way "full-duplex" MIMO relay. EURASIP J. Wirel. Commun. Netw. 2016(109), 1-16 (2016)

35. T Riihonen, S Werner, R Wichman, Mitigation of loopback self-interference in "full-duplex" MIMO relays. IEEE Trans. Signal Process. 59(12), 5983-5993 (2011) 
36. Y Wu, PA Chou, SY Kung, in Conf. on Information Sciences and Systems (CISS). Information exchange in wireless networks with network coding and physical-layer broadcast (Princeton, 2005)

37. R Zamir, Lattice Coding for Signals and Networks. (Cambridge University Press, Cambrige, 2014)

38. A Chockalingam, S Rajan, Large MIMO Systems. (Cambridge University Press, Cambrige, 2014)

39. A Sabharwal, P Schniter, D Guo, DW Bliss, S Rangarajan, R Wichman, In-band "full-duplex" wireless: challenges and opportunities. IEEE J. Sel. Areas Commun. 32(9), 1637-1652 (2014)

40. JS Lemos, F Rosário, FA Monteiro, J Xavier, A Rodrigues, in IEEE 16th International Workshop on Signal Processing Advances in Wireless Communications (SPAWC). Massive MIMO "full-duplex" relaying with optimal power allocation for independent multipairs (Stockholm, 2015), pp. 306-310

41. FA Monteiro, I Wassell, N Souto, MIMO Detection Methods. MIMO Processing for 4G and Beyond: Fundamentals and Evolution, chap. 2. (CRC Press, Boca Raton, 2014), pp. 47-117

42. SMR Islam, N Avazov, OA Dobre, KS Kwak, Power-Domain Non-Orthogonal Multiple Access (NOMA) in 5G Systems: Potentials and Challenges (2017). http://ieeexplore.ieee.org/document/7676258/

43. Z Ding, Y Liu, J Choi, Q Sun, M Elkashlan, I CL, HV Poor, Application of non-orthogonal multiple access in LTE and $5 G$ Networks. IEEE Commun. Mag. 55(2), 185-191 (2017)

44. S Liu, C Zhang, Non-orthogonal multiple access in a downlink multiuser beamforming system with limited CSI feedback. EURASIP J. Wirel. Commun. Netw. 2016, 239 (2016)

45. X Su, H Yu, W Kim, C Choi, D Choi, Interference cancellation for non-orthogonal multiple access used in future wireless mobile networks. EURASIP J. Wirel. Commun. Netw. 2016, 231 (2016)

46. T Riihonen, X Wang, in Relaying in "full-duplex" radio communication systems. Institution of Engineering and Technology (IET), (London 2016), pp. 129-173. Advanced Relay Technologies in Next Generation Wireless Communications, chap. 5

47. H Krishnaswamy, G Zussman, 1 Chip $2 x$ the bandwidth. IEEE Spectrum. 53(7), 38-54 (2016)

48. Z Zhang, X Chai, K Long, A Vasilakos, L Hanzo, Full duplex techniques for $5 G$ Networks: self-interference cancellation, protocol design, and relay selection. IEEE Commun. Mag. 53(5), 128-137 (2015)

49. ME Knox, in IEEE 13th Annual Wireless and Microwave Technology Conference (WAMICON). Single Antenna Full Duplex Communications using a Common Carrier (Cocoa Beach, 2012), pp. pp. 1-6

50. M Heino, D Korpi, T Huusari, Antonio-E Rodriguez, S Venkatasubramanian, T Riihonen, L Anttila, C Icheln, K Haneda, R Wichman, M Valkama, Recent advances in antenna design and interference cancellation algorithms for in-band full duplex relays. IEEE Commun. Mag. 53(5), 91-101 (2015)

51. I Krikidis, HA Suraweera, Full-duplex cooperative diversity with alamouti space-time code. IEEE Wirel. Commun. Lett. 2(5), 519-522 (2013)

52. HA Suraweera, I Krikidis, G Zheng, C Yuen, PJ Smith, Low-complexity end-to-end performance optimization in MIMO "full-duplex" relay system. IEEE Trans. Wirel. Commun. 13(2), 913-926 (2014)

53. M Duarte, Full-duplex Wireless: Design, Implementation and Characterization. PhD thesis, Rice University, 2012

54. H Alves, da DB Costa, RD Souza, M Latva-aho, in IEEE 14th Workshop on Signal Processing Advances in Wireless Communications (SPAWC). On the performance of two-way half-duplex and one-way "full-duplex" relaying (Darmstadt, 2013), pp. 56-60

55. P Lioliou, M Viberg, M Coldrey, F Athley, in 44th Asilomar Conference on Signals, Systems and Computers (ASILOMAR). Self-Interference Suppression in Full-Duplex MIMO Relays (Pacific Grove, 2010), pp. 658-662

56. E Antonio-Rodriguez, R Lopez-Valcarce, T Riihonen, S Werner, R Wichman, in IEEE 14th Workshop on Signal Processing Advances in Wireless Communications (SPAWC). Adaptive Self-Interference Cancellation in Wideband Full-Duplex Decode-and-Forward MIMO Relays (Darmstadt, 2013), pp. 370-374

57. F Rosário, FA Monteiro, A Rodrigues, Fast matrix inversion updates for massive MIMO detection and precoding. IEEE Signal Process. Lett. 23, 75-79 (2016)
58. DC Araújo, T Maksymyuk, ALF de Almeida, T Maciel, JCM Mota, M Jo, Massive MIMO: survey and future research topics. IET Commun. 10(15), 1938-1946 (2016)

59. U Madhow, D Brown, S Dasgupta, R Mudumbai, in Information Theory and Applications Workshop (ITA), 2014. Distributed Massive MIMO: Algorithms, Architectures and Concept Systems (San Diego, 2014), pp. 1-7

60. HA Suraweera, HQ Ngo, T Duong, C Yuen, E Larsson, in IEEE International Conference on Communications (ICC). Multi-Pair Amplify-and-Forward Relaying with Very Large Antenna Arrays (Budapest, 2013), pp. 4635-4640

61. TVT Le, YH Kim, Power and spectral efficiency of multi-pair massive antenna relaying systems with zero-forcing relay beamforming. IEEE Commun. Lett. 19(2), 243-246 (2015)

62. R Wyrembelski, T Oechtering, H Boche, in IEEE 19th International Symposium on Personal, Indoor and Mobile Radio Communications (PIMRC). Decode-and-Forward Strategies for Bidirectional Relaying (Cannes, 2008), pp. 1-6

63. T Koike-Akino, P Popovski, V Tarokh, Optimized constellations for two-way wireless relaying with physical network coding. IEEE J. Sel. Areas Commun. 27(5), 773-787 (2009)

64. J Zhan, B Nazer, M Gastpar, U Erez, in IEEE International Symposium on Information Theory (ISIT). MIMO Compute-and-Forward (Seoul, 2009), pp. 2848-2852

65. MM Molu, K Cumanan, A Burr, Low-complexity compute-and-forward techniques for multisource multirelay betworks. IEEE Commun. Lett. 20(5), 926-929 (2016)

\section{Submit your manuscript to a SpringerOpen ${ }^{\circ}$ journal and benefit from:}

- Convenient online submission

- Rigorous peer review

- Immediate publication on acceptance

- Open access: articles freely available online

- High visibility within the field

- Retaining the copyright to your article

Submit your next manuscript at $>$ springeropen.com 